\title{
Introduction to fractional linear systems. Part 2: Discrete-time case
}

\author{
M.D.Ortigueira
}

\begin{abstract}
In the paper, the class of discrete linear systems is enlarged with the inclusion of discrete-time fractional linear systems. These are systems described by fractional difference equations and fractional frequency responses. It is shown how to compute the impulse response and transfer function. Fractal signals are introduced as output of special linear systems: fractional differaccumulators, systems that can be considered as having fractional poles or zeros. The concept of fractional differaccumulation is discussed, gencralising the notions of fractal and $1 / f$ noise, and introducing two kinds of fractional differaccumulated stochastic process: hyperbolic, resulting from fractional accumulation (similar to the continuous-time case), and parabolic noise, resulting from fractional differencing.
\end{abstract}

\section{Introduction}

In a companion paper [1], we presented an introduction to fractional continuous-time linear systems. Such systems may be suitable for modelling several signals and systems found in practice. However, there are other similar situations where the continuous-time approach is not suitable; for example, in hydrology and economics there are time series with fractional characteristics that are not well fitted by the usual ARMA models [2]. The results obtained for continuous-time fractional systems have motivated us to consider the discrete-time counterpart. Although there are few current applications of fractional discrete-time systems, they are interesting and motivating enough for the development of the theory of such systems. The socalled fractional delay filter $[3,4]$ is a system for bandlimited interpolation between samples and is used in communications, speech processing, music technology, and array processing. In communications, fractionally spaced equalisers [5] became very popular, due to their performance. Attempts have been made to use fractional discrete-time systems in control [6-8] and economics $[2,9$, 10]. Here a combination of an ARMA model with a fractional differencing has been used (see also [11]).

These systems can be considered special cases of fractional discrete-time linear systems. In this paper, we present a generalisation of the usual theory to include these systems. Similarly to the situation verified previously [1], we find here that we are led to a formulation that looks like the usual formulation, leading to the impression that there is nothing new. However, that is not the case in practice, since to our knowledge there is no published work describing the systems we are considering here.

( IEE 2000

IEE Proceedings online no. 20000273

DOI: $10.1049 /$ ip-vis:20000273

Paper received 13th September 1999

The author is with the Instituto Superior Técnico and UNINOVA, Campus da FCT da UNL, Quinta da Torre 2825 - 114 Monte da Caparica, Portugal and also with INESC, R. Alves Redol, 9, $2^{\circ}, 1000$ Lisbon, Portugal
Usually discrete-time linear systems are described by difference equations, and characterised by their impulse responses and corresponding transfer functions and frequency responses. In the following we are concerned with the study of the linear systems described by fractional difference equations. These are similar to the usual, but admit non-integer delays or leads. For these equations we generalise the referred responses.

We begin by considering the definition of fractional delay and lead ('dellead'). We use the unit delay [4] with a discrete-time Fourier transform (FT) equal to $\mathrm{e}^{j(2) x}$, enabling us to generalise, again with the help of convolution, the usual translation property of the discrete-time FT. This, we define 'dellead' for signals with discrete-time FT. The next steps would lead us to try a similar definition concerning the dellead in the context of the $Z$ transform (ZT). Unfortunately, there is no causal or anti-causal sequence with ZT equal to $z^{x}(|z| \neq 1$ and $x \notin Z)$. This important fact forces us to use another method: the Cauchy integrals. These are operators that perform a projection of a function defined on the unit circle over the regions outside (casual case) or inside (anti-causal case) the unit circle. With these integrals we can treat the causal systems described by linear fractional difference equations, enabling us to define transfer function and impulse response. The developed theory is suitable for obtaining the modified ZT from the ZT [12].

We present an introduction of the fractional delay/lead (dellead). The treatment of the fractional delleads in the ZT context is carried out with the help of the Cauchy integrals, and examples are presented. We also study the linear systems described by fractional difference equations, and introduce the notions of fractional transfer function and impulse response. We use the partial fraction expansion, and treat the cases corresponding to rational and irrational delleads. Stability is also studied. The initial condition problem is discussed, and the results are used later, when we introduce a state-space formulation. Fractal signals [14, 15] are introduced as output of special linear systems: fractional differaccumulators, systems that can be considered as having fractional poles or zeros. The concept of fractional differaccumulation is also discussed. 


\section{Fractional delay/lead of discrete-time signals}

\subsection{Fractional delay/lead}

In practical applications of discrete systems, mostly we deal with signals that are sampled versions of continuoustime band-limited signals. Normally, these signals are processed synchronously, in the sense that the time domain is the set of integer numbers for all of them. However, there are applications where this does not happen. We can process signals obtained by sampling continuous-time signals with the same sampling interval but in different instants of time, e.g. the so-called timedelayed processes [6]. On the other hand, in other applications, we may need to know the behaviour of a system between the sampling instants [12]. The current application of multi-rate techniques allows the conversion of a signal sampled with a given sampling interval to another one with a different sampling interval. These considerations are intended to be a motivation for the theory we are going to develop. The notions of 'delay' and 'lead' are usually defined only for integer multiples of a given time interval. Essentially, and by analogy with fractional differintegration, we intend to generalise these notions to allow for fractional delay and lead (dellead). In Table 1 we present a relation between integer delleads and powers of $z$.

We intend to prolong the sequences to give sense to $\delta_{n-z}$ (To make the theory similar to that developed for continuous-time systems, we use here the Kronecker $\delta$ instead of Dirac $\delta(t)$. To avoid confusion, we use the notation $\delta^{(\alpha)}(t)$ to represent the $\alpha$-order differintegration of Dirac $\delta(t)$ and $\delta_{n-\alpha}$ to represent the fractional translation of the Kronecker $\delta_{n}$ ) and $z^{-x}$. We assume $\alpha$ to be any non-integer real. If $\alpha<0$, we have a fractional lead; if $\alpha>0$, we have a fractional delay. When $\alpha$ is integer, we fall into the usual scheme.

We are looking for sequences, $\delta_{n-x}$ : bilateral (with $\mathrm{e}^{-j \omega x}$ as FT) or causal, and anti-causal (with $z^{-\alpha}$ as the $Z$-transform). For this, we have tried to compute the inversion integral using the theory of complex variable functions. The function $z^{-\alpha}$ has a branch point at $z=0$. Any branch cut will cross the unit circle but will not serve to define two regions like $|z|<1$ and $|z|>1$. Therefore, we cannot impose causality by the choice of the branch cut, in contrast to the continuous-time case [14]. On the other hand, if $\alpha$ is not integer, $z^{-\alpha}$ with $|z| \neq 1$ does not have a convergent MacLaurin or Laurent expansion, which implies that there is no causal nor anti-causal sequence with $z^{-\alpha}$ as ZT. The bilateral case is very simple: it is enough to compute the inverse discrete-time Fourier transform, $h_{\alpha}(n)$, of $\mathrm{e}^{-j \omega x}$. It is readily computed and is given by

$$
h_{\alpha}(n)=\frac{\sin [\pi(n-\alpha)]}{\pi(n-\alpha)}=\frac{\sin (\pi \alpha)}{\pi \alpha} \frac{(-1)^{n}}{1-\frac{n}{\alpha}} \quad n \in Z
$$

Defining a FT $X_{\alpha}\left(\mathrm{e}^{j \omega}\right)$ by

$$
X_{\alpha}\left(\mathrm{e}^{j \omega}\right)=\mathrm{e}^{-j \omega x} X\left(\mathrm{e}^{j \omega}\right)
$$

and putting $x_{n-\alpha}$ as the inverse FT of $X_{y}\left(\mathrm{e}^{j(t)}\right)$

$$
X_{\alpha}\left(\mathrm{e}^{j \omega}\right)=\sum_{-\infty}^{\infty} x_{n-\alpha} \mathrm{e}^{-j n \omega}
$$

Table 1: Integer delleads

\begin{tabular}{lllllllllll}
\hline$\ldots$ & $z^{-k}$ & $\ldots$ & $z^{-2}$ & $z^{-1}$ & 1 & $z$ & $z^{2}$ & $\ldots$ & $z^{k}$ & $\ldots$ \\
\hline$\ldots$ & $\delta_{n-k}$ & $\ldots$ & $\delta_{n-2}$ & $\delta_{n-1}$ & $\delta_{n}$ & $\delta_{n+1}$ & $\delta_{n+2}$ & $\ldots$ & $\delta_{n+k}$ & $\ldots$ \\
\hline
\end{tabular}

we conclude that

$\left.x_{n-\alpha}=\sum_{m=-\infty}^{+\infty} x_{m} \frac{\sin [\pi(n-\alpha-m)]}{\pi[n-\alpha-m]} \quad \alpha \in\right] 0,1[\quad n \in Z$

(note that this formula remains valid for any real $\alpha$.)

Eqn. 3 defines the modified Fourier transform [12] that is a special case of the Zak transform [13] and generalises the translation property of the FT. Eqn. 4 (essentially the Shannon-Whitaker interpolation formula) is very important, since it states a relation between two signals $x_{n}$ and $y_{m}=x_{n+x}$ defined in the sets $Z$ and $\{m: m=n+x, n \in Z$ and $x \in R\}$, respectively. Thus, we are relating two signals defined over two time grids, obtained one from the other by a fractional translation (note that this formula remains valid for any real $\alpha$ ). The relation in eqn. 4 is a convolution between $x_{n}$ and $h_{\gamma}(n)$, which can be considered as the impulse response of a reconstruction filter that is noncausal and IIR; a review of FIR and all-pass filter design techniques for bandlimited approximation of this filter is presented elsewhere [4], together with several applications.

According to the properties of the convolution and to the fact that $h_{\gamma}(n)$ is square summable, it is enough that $x_{n}$ is also square summable to guarantee that the series in eqn. 4 is convergent. Using Fourier transform theory, we can show that this relation remains valid if $x_{n}$ is an absolutely summable signal.

With eqns. 2-4 we are ready to define fractional difference equations and to introduce a frequency response for the systems represented by such equations.

According to the notation used in Table 1 , we can substitute $h_{y}(n)$ for $\delta_{n-\gamma}$ :

$$
\delta_{n-\alpha}=h_{\alpha}(n) \text { if } \alpha \text { non-integer }
$$

Thus, we can write eqn. 4 in the form

$$
D_{0}^{\alpha} x_{n}=x_{n-\alpha}=x_{n} * h_{\varkappa}(n)=x_{n} * \delta_{n-\mu}
$$

This is analogous to the differintegration of a continuoustime signal [1].

Continuing our sequence of thoughts, we look for the causal and anti-causal solutions for fractional delleads. We would like to generalise eqn. 2 for signals with ZT:

$$
X_{y}(z)=z^{-\mathcal{x}} X(z) \quad|z|>1 \text { or }|z|<1
$$

This fact means that we were looking for the impulse response of a filter with transfer function equal to $z^{x}$ (it is interesting to note that $X_{\gamma}(z)$ is the modified ZT [12]). However, as previously concluded [1], there is no such filter. Therefore, to obtain $X_{\alpha}(z)$, we perform analytic continuations of a function $g(z)$ defined on the unit circle $\left(z=\mathrm{e}^{j(\omega x}\right)$ to the region $|z|>1\left(G^{+}(z)\right)$ and $|z|<1\left(G^{-}(z)\right)$, in such a way that their projections on the space corresponding to that circle satisfy eqn. 92 (see Appendix):

$$
G^{+}(z)+G^{-}(z)=g(z) \quad \text { for }|z|=1
$$

The extrapolations are obtained through the use of the Cauchy's integrals (see Appendix), We obtain the ZT of the causal and anti-causal parts of a given signal, from its Fourier transform. As shown in the Appendix

$$
\begin{aligned}
& G^{+}(z)=\frac{1}{2 \pi j} \int_{L} \frac{g(w) \cdot w^{-1}}{1-w z^{-1}} d w \\
& =\frac{1}{2 \pi} \int_{-\pi}^{\pi} \frac{g\left(\mathrm{e}^{j \omega}\right)}{1-\mathrm{e}^{j \omega} z^{-1}} \mathrm{~d} \omega \quad|z|>1
\end{aligned}
$$


and

$$
\begin{aligned}
G^{-}(z) & =\frac{1}{2 \pi j} \int_{L} \frac{g(w) \cdot z \cdot w^{-1}}{w-z} \mathrm{~d} w \\
& =\frac{1}{2 \pi} \int_{-\pi}^{\pi} \frac{g\left(\mathrm{e}^{j(\omega)}\right) \cdot z}{\mathrm{e}^{j(\omega}-z} \mathrm{~d} \omega \quad|z|<1
\end{aligned}
$$

$L$ is the integration path: the unit circle. In fact, let $X(w)$ be given by

$$
X(w)=\sum_{-\infty}^{\infty} x_{n} w^{-n} \quad \text { with } w=\mathrm{e}^{j \omega}
$$

and

$$
\frac{1}{1-w z^{-1}}=\sum_{0}^{\infty} w^{n} \cdot z^{-n} \quad|z|>1
$$

From eqn. 9 we obtain

$$
X^{+}(z)=\sum_{0}^{\infty} x_{n} z^{-n}
$$

For the anti-causal case, the situation is similar:

$$
X^{-}(z)=\sum_{-\infty}^{-1} x_{n} z^{-n}
$$

The use of eqns. 13 and 3 allows us to obtain

$$
X_{\alpha}^{+}(z)=\sum_{0}^{\infty} x_{n-\alpha} z^{-n}=\sum_{0}^{\infty} x_{n} * \delta_{n-\alpha^{2}} z^{-n}
$$

For the anti-causal case, the procedure is similar:

$$
X_{\alpha}^{-}(z)=\sum_{-\infty}^{-1} x_{n-\alpha^{-}} z^{-n}=\sum_{-\infty}^{-1} x_{n} * \delta_{n-\alpha} z^{-n}
$$

These results lead us to introduce formally the modified ZT by

$$
X_{\alpha}(z)=X_{\alpha}^{+}(z)+X_{\alpha}^{-}(z)
$$

This means that if a given signal has a ZT, it also has a modified ZT. However, there is no operator to allow us to obtain directly the modified ZT from the ZT, or vice versa.

\section{Discrete-time fractional linear systems}

\subsection{Difference fractional equations}

In this Section, we generalise the concept of difference equation in order to consider non-integer delays or leads. Consider the linear time-invariant systems characterised by a difference equation that adopts the general form

$$
\sum_{i=0}^{N_{0}} a_{i} D^{v_{i}} y(n)=\sum_{j=0}^{M_{0}} b_{j} D^{v_{j}} x(n) \quad n \in Z
$$

where $\mathrm{D}$ is the difference operator (delay) and the $v_{n}$ are the delays that, in the general case, are real numbers. This means that we made $\mathrm{D}^{v_{n}} y(t)=y\left(t-v_{n}\right)$. According to our considerations in Section 2 the relation $\mathrm{D}^{v_{i}} z^{n}=z^{-v_{i} z^{n}}$ remains valid only for $|z|=1$; in this case, we obtain, easily

$$
H(z)=\frac{\sum_{j=0}^{M_{0}} b_{j} z^{-v_{j}}}{\sum_{i=0}^{N_{0}} a_{i} z^{-v_{i}}} \quad z=\mathrm{e}^{j \omega}
$$

This is a frequency response function. Again, according to our earlier considerations, we can obtain the transfer functions, corresponding to causal and anti-causal systems by using the Cauchy integrals for projecting $H(z)$ in eqn. 19 defined on the unit circle outside and inside it. To obtain the impulse response, we must perform the inversion of the transfer function. As in the continuous-time case, in the following we consider that the $v_{n}$ are either rational numbers or are multiples of a common real, v. Eqns. 18 and 19 then adopt the format

$$
\sum_{i=0}^{N} a_{i} D^{-i v} y(n)=\sum_{j=0}^{M} b_{j} D^{-j v} x(n)
$$

and

$$
H(z)=\frac{\sum_{j=0}^{M} b_{j} z^{-j v}}{\sum_{i=0}^{N} a_{i} z^{-i v}} \quad z=\mathrm{e}^{j(s)}
$$

We use the term 'fractional autoregressive moving average (FARMA) system'. In contrast to the continuous-time case, we must consider two situations; the general FARMA system and the particular (fractional MA - FMA). This latter case is very easy to deal with, because $H\left(\mathrm{e}^{j \omega}\right)$ is given by

$$
H\left(\mathrm{e}^{j(\omega)}\right)=\sum_{m=0}^{M} b_{m} \mathrm{e}^{-j m \omega v}
$$

we only have to use eqn. 9 to obtain $H^{+}(z)$. We have

$$
H^{+}(z)=\frac{1}{2 \pi} \int_{-\pi}^{\pi} \frac{\sum_{m=0}^{M} b_{m} \mathrm{e}^{-j m \omega \mathrm{V}}}{1-\mathrm{e}^{j \omega} z^{-1}} \mathrm{~d} \omega \quad|z|>1
$$

and

$$
\begin{aligned}
H^{+}(z) & =\sum_{m=0}^{\infty} z^{-n} \sum_{m=0}^{M} b_{m} \frac{1}{2 \pi} \int_{-\pi}^{\pi} \mathrm{e}^{j \omega(n-m v)} \mathrm{d} \omega|z|>1 \\
& =\sum_{m=0}^{\infty} \mathrm{z}^{-n} \sum_{m=0}^{M} b_{m} \delta_{n-m v}
\end{aligned}
$$

Thus, the impulse response is

$$
h(n)=\sum_{m=0}^{M} b_{m} \delta_{n-m \mathrm{v}} \quad n \geq 0
$$

If $v=1 / q$

$$
h(n)=\sum_{k=0}^{\lfloor M / q\rfloor} \sum_{l=0}^{q-1} b_{k q+l} \delta_{n-k-l v} \quad n \geq 0
$$

The symbol $\lfloor$.$\rfloor means integer part. If q=1$, we obtain the usual response corresponding to a FIR system.

Next we consider the general FARMA case. Without restrictions, we may assume that the fraction in eqn. 21 is proper. The computation of the impulse response follows steps similar to the continuous-time case.

(a) Consider the function $H(w)$, by substitution of $w$ for $z^{y}$. (b) The polynomial denominator in $H(w)$ is the indicial polynomial or characteristic pseudo-polynomial. Perform the expansion of $H(w)$ into partial fractions such as

$$
F(w)=\frac{1}{\left(1-a \cdot w^{-1}\right)^{k}}
$$


(c) Substitute back $z^{\prime}$ for $w$ to obtain $H(z)$ expanded as a linear combination of fractions such as

$$
F(z)=\frac{1}{\left(1-a \cdot z^{-1}\right)^{k}}
$$

(d) Compute the impulse responses corresponding to each partial fraction.

(e) Add the impulse responses.

These steps allow us to test the stability of fractional linear time-invariant discrete-time systems, according to how we obtain the impulse response. In fact, we have transformed the denominators in rational functions of $w^{-1}$. The properties of the response corresponding to each partial fraction depend on the poles, as in the non-fractional case. Thus, to test the stability of the fractional linear systems, we only have to check if the poles are outside the unit circle.

\subsection{Partial fraction inversion}

3.2.1 Rational case: We proceed to the inversion of the partial fraction (eqn. 28). Let us consider the case where $k=1$ and $v=1 / q$. The problem resumes to the computation of the inverse ZT of

$$
F^{+}(z)=\frac{1}{2 \pi \mathrm{j}} \int_{l_{l}} \frac{1}{1-a \cdot v^{-1}} \frac{v^{-1}}{1-v z^{-1}} \mathrm{~d} v \quad|z|>1
$$

For $v=\mathrm{e}^{j \omega}$, we have

$$
F^{+}(z)=\frac{1}{2 \pi} \int_{-\pi}^{\pi} \frac{1}{1-a \cdot \mathrm{e}^{-j \omega y}} \frac{1}{1-\mathrm{e}^{j(\omega)} z^{-1}} \mathrm{~d} \omega \quad|z|>1
$$

The procedure that is followed is almost identical to that used in the continuous-time case [1] and consists of transforming the partial fraction into another equivalent one:

$$
\frac{1}{1-a \cdot \mathrm{e}^{-j \omega \omega}}=\frac{\sum_{i=0}^{q-1} a^{i} \cdot \mathrm{e}^{-j \omega \mathrm{i} r}}{1-a^{q} \mathrm{e}^{-j \omega}}
$$

For the proof, it is enough to sum the numerator on the right-hand side. If $|a|<1$, we can rewrite eqn. 31 in the form

$$
\frac{1}{1-a \cdot \mathrm{e}^{-j(\omega)}}=\sum_{n=0}^{\infty} \sum_{i=0}^{q-1} a^{q n+i} \mathrm{e}^{-j \omega(n+i v)}
$$

We thus conclude that the inverse ZT of $F^{+}(z), f(n)$, is given by

$$
f(n)=\sum_{i=0}^{q-1} a^{i} \sum_{m=0}^{\infty} a^{q m} \delta_{n-m-i v} \quad n \geq 0
$$

It is interesting to note that this case can be obtained from eqn. 26 with $M=\infty$ and setting $b_{m}$ an exponential sequence. The result (eqn. 31) states that if $v$ is rational, the general FARMA system has another equivalent description in terms of a transfer function with a rational denominator. In addition, eqns. 31 and 33 lead us to conclude that the computed impulse response is, in fact, a weighted average $\left(a^{i}, i=0, \ldots, q-1\right.$, as weights $)$ of $q$ responses of a first-order system to impulses applied at $-i v(i=0, \ldots, q-1)$.

In the $k>1$ case, the convolution property can be used, or we can relate eqn. 28 with the $(k-1)$ th derivative of $1 /\left(1-a \cdot z^{-1}\right)$ and use the differentiation property of the FT.
3.2.2 Irrational case: Consider now the case corresponding to $v$ as an irrational number. We manipulate eqn. 32 directly. Assume for now that $|a|<1$. In this case, we have

$$
\frac{1}{1-a \cdot \mathrm{e}^{-j \omega)}}=\sum_{n=0}^{\infty} a^{n} \mathrm{e}^{-j(\omega n n}
$$

Proceeding as in the previous case, we obtain

$$
f(n)=\sum_{k=0}^{\infty} a^{k} \cdot \delta_{n-k,} \quad n \geq 0
$$

This is the discrete analogue to the Mittag-Leffer function [1]. To test the coherence of this result, make $v$ a rational number: $v=1 / q$ in eqn. 34 . Now put $n=l \cdot q+i(l=0,1$, $2, \ldots ; i=0,2, \ldots, q-1)$ in the summation symbol to obtain

$$
F(\omega)=\sum_{l=0}^{\infty} a^{l q} \cdot \mathrm{e}^{-j \omega t} \sum_{i=0}^{q-1} a^{i} \cdot \mathrm{e}^{-j \omega i / q}
$$

This leads to eqn. 32. With a similar substitution in eqn. 35 , we are led to eqn. 33 again.

3.2.3 Practical example: The Unit delay operator has been studied previously [4], and several approaches to implement causal approximations for it proposed. Here, we propose an alternative based on the theory we have presented: the fractional linear prediction.

Let $x(n) n \in Z$ be a real stationary stochastic process, observed up to time $n-1$, and let $R_{x}(k)$ be its autocorrelation function. We define the $N$ th order $d$-step prediction at $n-1+d(d \in R)$ (in fact, if $d<0$, we are making an interpolation, not a prediction) by

$$
\hat{x}(n-1+d)=-\sum_{i=1}^{N} a_{i} x(n-i)
$$

where $a_{i}(i=1, \ldots, N)$ are the coefficients of the $d$-step predictor (with $d=1$, we obtain the usual one-step prediction). The predictor coefficients are chosen in order to minimise the prediction error power:

$$
P_{d}=E\left[(x(n-1+d)-\hat{x}(n-1+d))^{2}\right]
$$

Assuming that the correlation matrix has rank at least equal to $N$, the minimisation of the prediction error power (eqn. 38 ) is easily performed by differentiation of its right-hand side in order to all the $a_{i}(i=1, \ldots, N)$, and leads to the following set of normal equations:

$$
\sum_{i=1}^{N} a_{i}, R(k-i)=-R(-k-d+1) \quad k=1,2, \ldots, N
$$

whence we obtain the predictor coefficients. As an application example, we performed the computation of the predictor coefficients for $x_{n}=\cos \left(\omega_{0} n\right)$. The predictor is given by

$$
\left[\begin{array}{l}
a_{1} \\
a_{2}
\end{array}\right]=-\frac{\sin \left(\omega_{0}\right)}{1-\cos ^{2}\left(\omega_{2}\right)}\left[\begin{array}{c}
-\sin \left[\omega_{0}(d+1)\right] \\
\sin \left(\omega_{0} d\right)
\end{array}\right]
$$

and

$$
x_{n+d}=\cos \left[\omega_{0}(n+d)\right]
$$

as expected. It is a simple matter to implement this algorithm in a lattice form. In addition, the predictor coefficients can be adaptively computed.

IEE Proc.-Vis. Image Signal Process., Vol. 147, No. 1, February 2000 


\section{Results on fractional systems}

\subsection{Initial value problem}

In ordinary linear systems, either continuous-time or discrete-time, the insertion of the initial conditions when they are not zero is very simple, since the one-sided Laplace and $Z$ transforms make them appear explicitly. The same happens with the fractional continuous-time systems [1]. However, this does not happen here, because, in general, the number of initial values is not finite. We do not consider the problem with all generality. We consider a simple case for insight into the solution of the state variable equations.

Assume a continuous-time difference equation:

$$
x(t+\alpha)=x(t) \quad \alpha>0, t>0
$$

As known, the solution is the causal periodic repetition of a given initial function $x_{0}(t)$ defined in the interval $[0, \alpha[$. Thus

$$
x(t+n \alpha)=x_{0}(t) \quad t \in[0, \alpha[\quad \text { and } n=0,1,2, \ldots
$$

Consider a point $t_{0} \in[0, \alpha$. This point may be considered the origin of a sequence

$$
t_{n \vartheta}=t_{0}+n \alpha \quad n=1,2, \ldots
$$

where $x(t)$ assumes the same value, $x\left(t_{0}\right)$. Let $T \neq \alpha$ and consider another sequence

$$
t_{k T}=k \cdot T \quad T \in R \text { and } k=1,2, \ldots
$$

Consider the set of points belonging to both sequences. It is evident that, in general, their intersection is empty. A necessary and sufficient condition for non-empty intersections is that $t_{0}, T$, and $\alpha$ are commensurate:

$$
\frac{T}{\alpha}=\frac{q}{p},(p \neq q) \text { and } t_{0}=\varepsilon_{0} \cdot \alpha=\frac{r}{p} \cdot \alpha \quad(0 \leq r<p)
$$

where $p, q$, and $r$ are positive integers. In this case, for a given integer $k$, there is one $s \in\{0,2, \ldots, p-1\}$ such that

$$
n=k \frac{q}{p}+\frac{s}{p}
$$

It is clear that the intersection set has infinite cardinality. In fact, if the pair $(n, k)$ verifies eqn. 47 , all the pairs $(n+m q$, $k+m p)$ with $m=0,1,2, \ldots$ satisfy eqn. 46 and $x[(k+m p) T)$ assumes the constant value $x_{0}(r / p, \alpha)$, $r \in\{0,1,2, \ldots, p-1\}$. Considering all the values assumed by $r$, we conclude that we will obtain a periodic sequence $x(k T)$ with period $p$. Therefore, we need $p$ initial conditions.

Let us proceed a little further by considering

$$
x(t+\alpha)=a \cdot x(t)
$$

Let $X(s)$ be the unilateral Laplace transform of $x(t)$. Apply it to eqn. 48. After some easy manipulation, we obtain

$$
X(s)=\frac{\int_{0}^{\alpha} x(\tau) \mathrm{e}^{-s \tau} \mathrm{d} \tau}{1-a \mathrm{e}^{-s \alpha}} \operatorname{Re}(s)>0
$$

If $|a|<1$ and $x(t)=x_{0}(t)$ for $t \in[0, \alpha[$,

$$
X(s)=\sum_{n=0}^{\infty} a^{n} \mathrm{e}^{-s n \gamma} \cdot \int_{0}^{\alpha} x_{0}(\tau) \mathrm{e}^{-s \tau} \mathrm{d} \tau \quad \operatorname{Re}(s)>0
$$

leading to

$$
x(t)=a^{n} x_{0}(t-n \alpha) \quad t \in[n \alpha,(n+1) \alpha[\quad \text { and } n=1,2, \ldots
$$

We conclude that this situation is similar to the previous one, but now the replications of the initial value $x_{0}(s / p \cdot \alpha)$ are weighted by $a^{n}$. Putting $t=n \alpha+\varepsilon_{0}$, $\alpha$ with $0 \leq \varepsilon_{0}<1$, it becomes

$$
x\left[n \alpha+\varepsilon_{0} . \alpha\right]=a^{n} x_{0}\left[\varepsilon_{0} . \alpha\right] \quad n=1,2, \ldots
$$

and from eqn. 46 ,

$$
\begin{aligned}
x[n p / q \cdot T+r / q \cdot T]= & a^{n} x_{0}[r / q \cdot T] \\
& 0 \leq r<p ; n=1,2, \ldots
\end{aligned}
$$

The integer $r$ must be chosen in order to lead to an integer value for the expression

$$
k=n p / q+r / q
$$

Denote by $r_{k}$ the sequence of residuals obtained by dividing $k \cdot q$ by $p$. This sequence is periodic with period $p$. On the other hand, when $k$ is increased by $p, n$ is incremented by $q$. Thus

$$
x[(k+p) . T]=a^{q} x(k \cdot T)
$$

The $Z$ transform gives

$$
X(z)=\frac{\sum_{i=0}^{p-1} x(i \cdot T) z^{-i}}{1-a^{q} \cdot z^{-p}}
$$

where the initial values $x(i . T)$ belong to the set $\left\{x_{0}(r \cdot T)\right.$, $0 \leq r<p\}$. The order of appearance depends on $p$ and $q$. For example, assume that $p=4$ and $q=7$ and, for simplicity, $T=1$. In this case, $x(1)=x_{0}(3 / 7), x(2)=x_{0}(2 / 7)$, $x(3)=x_{0}(1 / 7)$, and $x(4)=x_{0}(0)$. From eqn. 51 and making $t=k-T$, we obtain

$$
x(k T)=a^{n} x_{0}(\zeta) \quad k T \in[n \alpha,(n+1) \alpha[\quad \text { and } k=1,2, \ldots
$$

where $\zeta$ is the residual of the division of $k T$ by $\alpha$. Of course, $n$ is the quotient: $n=\lfloor k T / \alpha\rfloor$. If $T / \alpha$ is not rational, the sequence $r_{k}$ defined above is aperiodic, and so we need an infinite number of initial values to define $x(k-T)$ for $k=1,2, \ldots$

\subsection{State variable representation}

The state-space representation of a fractional linear system described by eqn. 20 is readily obtained and can be represented by

$$
\boldsymbol{s}(k+\alpha)=\boldsymbol{A s}(k)+\boldsymbol{B} \boldsymbol{x}(k)
$$

and

$$
\boldsymbol{y}(k)=\boldsymbol{C s}(k)+\boldsymbol{D} \boldsymbol{x}(k)
$$

where $s(t)$ is the state vector. We do not perform the computation of the solution of eqns. 58 and 59 , only to solve the homogeneous equation

$$
\boldsymbol{s}(k+\alpha)=\boldsymbol{A} \cdot \boldsymbol{s}(k)
$$

Assume that $\boldsymbol{A}$ is a non-singular matrix. According to Section 4.1, it is not hard to show that the solution for eqn. 60 is easily obtained from eqn. 56. However, that result is not compatible with the state variable formulation. We must have only one initial state. To avoid the problem, we have to assume that all the initial conditions are equal to 
a given value, $s_{0}$. In this case, the solution of eqn. 60 is given by

$$
\boldsymbol{s}(k)=\boldsymbol{\Phi}(k, 0) \boldsymbol{s}(0) \quad k>0
$$

where, again, $n$ is equal to $\lfloor k T / \alpha\rfloor$ and $s(0)$ is a vector with all the components equal to a given constant, $s_{0}$.

As in the ordinary case, we have a state transition operator, given by

$$
\Phi(k, 0)=A^{k} \quad k>0
$$

This seems to suggest that, in the time-variant case, the state transition operator must be given by

$$
\Phi(k, 0)=\prod_{i=1}^{n} A(i) \quad(k>0)
$$

We do not pursue the study of this operator. However, we point out that it is not very difficult to see that, in general and according to the results of Section 4.1, it does not satisfy the semi-group property enjoyed by the ordinary state transition operator.

Having solved the homogeneous equation (eqn. 60), we are going to solve eqn. 58 , under null initial conditions. This is readily done by the use of the theory developed in Sections 2.1 (eqns. 8 to 10) and 3.2 (eqn. 19). From eqn. 58 we obtain

$$
\left[\mathrm{e}^{j \omega a} \boldsymbol{I}-\boldsymbol{A}\right] \boldsymbol{S}\left(\mathrm{e}^{j \omega}\right)=\boldsymbol{B} \cdot X\left(\mathrm{e}^{j \omega}\right)
$$

Assuming that the determinant of $\boldsymbol{A}$ is less than one, we can write

$$
\boldsymbol{S}\left(\mathrm{e}^{j \omega}\right)=\sum_{n=0}^{\infty} \boldsymbol{A}^{n} \cdot \boldsymbol{B} \cdot \mathrm{e}^{-j \omega(n+1) \alpha} \cdot X\left(\mathrm{e}^{j \omega)}\right)
$$

With the help of the Cauchy integrals (Section 2.1), we obtain

$$
\boldsymbol{s}(k)=\sum_{n=0}^{\infty} \boldsymbol{A}^{n} \cdot \boldsymbol{B} \cdot x[k-(n+1) x] \quad k \geq 0
$$

This shows that, to compute the state at instant $t=k T$, in addition to the current input value, we must know all the past values at instants $t=k T-(n+1) \alpha T$. Note that these values are computed from all the values of the input at $t=k T$ with $k=0,1, \ldots, \infty$, unless we can use the algorithm proposed in Section 4.1.

\section{Fractional order pole or zero}

In Section 3, we studied fractional linear systems having transfer functions with expansions in partial fractions with the general format (eqn. 28). As shown if $v=1 / q$, with $q$ a positive integer, these fractions can be transformed into others with a non-fractional denominator. Thus, eqn. 28 corresponds to a fraction with a $k$-order pole. Now we generalise these results by introducing the notions of fractional order pole, and similarly fractional order zero. In terms of systems, we consider systems with transfer functions

$$
H(z)=\left(1-a \cdot z^{-1}\right)^{y}
$$

corresponding to a $v$-order zero if $v>0$, and to a $v$-order pole if $v<0$. At a first glance, we would say that these systems are useless. However, the special case obtained by putting $a=1$ in eqn. 67 is very important. It may be called the differaccumulator; fractional differencer if $v>0$, and accumulator (or integrator) if $v<0$. These terms have been used in fractal modelling $[14,15]$ and $1 / f$ noise $[16,17]$. In economics and hydrology [2,9-11], a combination of an ARMA system and a differaccumulator has been used to model some experimental signals. The difference equation corresponding to eqn. 67 can be described by a fractional order difference equation:

$$
(1-a \cdot D)^{r} y_{n}=x_{n} \text { or } y_{n}=(1-a \cdot D)^{n} x_{n}
$$

where $\mathrm{D}$ is the unit delay operator. The corresponding impulse response is obtained from the coefficients of the binomial series. Note only that, for $|z|>|a|$

$$
\left(1-a \cdot z^{-1}\right)^{r}=\sum_{k=0}^{\infty}(-1)^{k}\left(\begin{array}{l}
y \\
k
\end{array}\right) a^{k} z^{-k}
$$

The corresponding impulse response is

$$
h_{n}=(-1)^{n} a^{n}\left(\begin{array}{l}
v \\
n
\end{array}\right) u_{n}
$$

The series is absolutely and uniformly convergent for $|z|>|a|$. Essentially, eqn. 67 corresponds to the generalisation of the notions of finite difference $\{a=1$ and $v=n\}$, $\left(1-z^{-1}\right)^{n}$ and of accumulation $\{a=1$ and $v=-n\}$ $\left(1-z^{-1}\right)^{-n}$. Alternatively, we can obtain another expression by using

$$
\left(1-a \cdot z^{-1}\right)^{y}=\frac{1}{\left(1-a \cdot z^{-1}\right)^{-1}}=\frac{1}{\sum_{k=0}^{\infty}(-1)^{k}\left(\begin{array}{c}
-1 \\
k
\end{array}\right) z_{0}^{k} z^{-k}}
$$

This means that the system with transfer function $H(z)=\left(1-a \cdot z^{-1}\right)^{r}$ can be considered as an infinite order AR or MA system. This is a very singular and interesting case. We are interested in the special case when $a=1$, fractional differencing $(v>0)$ or fractional accumulation $(v<0)$.

Regarding the stability of the system $\left(1-z^{-1}\right)^{y}$, we can say that

(i) if $v$ is a negative integer, it is unstable in general, except the case when $v=-1$ (accumulator): in this situation. the system is wide-sense stable.

(ii) if $y$ is a positive integer, it is a FIR, and so it is always stable in every sense.

(iii) according to results presented elsewhere [18], the series $\Sigma_{0}^{+\infty}\left|h_{n}\right|$ converges for every $v>0$, but not for $v<0$ : if $v>0$, the system will be stable in any sense; if $-1<v<0$, the system will be wide-sense stable, since the impulse response goes slowly to zero, $h_{n} \approx c \cdot n^{-v-1}$ :

$$
\left(\begin{array}{l}
v \\
k
\end{array}\right)=\frac{(-1)^{k}}{\Gamma(-1 \cdot)} \cdot \frac{\Gamma(k-v)}{\Gamma(k+1)}
$$

and

$$
\frac{\Gamma(k-v)}{\Gamma(k+1)}=c \cdot k^{-v-1}
$$

where $c$ is a positive constant, $h_{n} \approx c \cdot n^{-v-1}$.

We conclude that if $|v|<1$, the system is at least widesense stable and has two wide-sense stable representations (MA or AR).

As before, we define the fractional (discrete-time) stochastic process as the output of a fractional system excited by stationary white noise. Let $h(k)$ be the impulse response of the system. Therefore, a fractional stochastic process is

$$
x(k)=h(k) * n(k)
$$


where $n(k)$ is a stationary white noise with variance $\sigma^{2}$. We define, as usual, the autocorrelation function and power spectral density (spectrum) by

$$
R_{x}(k)=\sigma^{2} \sum_{i=0}^{\infty} h_{i}^{*} h_{i+k} \quad k \geq 0
$$

and

$$
S_{x}\left(\mathrm{e}^{j \omega}\right)=\sigma^{2} \cdot\left|H\left(\mathrm{e}^{j \omega}\right)\right|^{2}
$$

According to eqn. 74, we define an $\alpha$-order fractionally differaccumulated noise as the output of the system with impulse response given by eqn. 70, with $a=1$, when the input is stationary white noise.

The computation of its autocorrelation function is slightly complex. Eqns. 70 and 75 allow us to obtain the autocorrelation function. Inserting eqn. 70 into eqn. 75, we obtain

$$
R_{x}(k)=\sigma^{2} \sum_{i=0}^{\infty}(-1)^{i}\left(\begin{array}{c}
\alpha \\
i
\end{array}\right)(-1)^{i+k}\left(\begin{array}{c}
\alpha \\
i+k
\end{array}\right) \quad k \geq 0
$$

or

$$
R_{x}(k)=\sigma^{2}(-1)^{k} \sum_{i=0}^{\infty}\left(\begin{array}{c}
\alpha \\
i
\end{array}\right)\left(\begin{array}{c}
\alpha \\
i+k
\end{array}\right) \quad k \geq 0
$$

Let us introduce the Gauss hypergeometric function [18]:

$$
{ }_{2} F_{1}(a, b ; c ; z)=\sum_{k=0}^{\infty} \frac{(a)_{k}(b)_{k}}{(c)_{k}} \cdot \frac{z^{k}}{k !}
$$

where $c \neq 0,-1,-2, \ldots$ and $(a)_{k}$ is the Pochhammer symbol: $(a)_{k}=a \cdot(a+1)(a+2) \ldots(a+k-1)$. The series in eqn. 79 is convergent for $|z| \leq 1$, if $c-a-b>0$ :

$$
\begin{aligned}
& { }_{2} F_{1}(a, b ; c ; z) \\
& \quad=\frac{\Gamma(c)}{\Gamma(b), \Gamma(c-b)} \int_{0}^{1} t^{b-1}(1-t)^{c-b-1}(1-z t)^{-a} \mathrm{~d} t
\end{aligned}
$$

if $0<b<c$ and $|\arg (1-z)|<\pi$, that function can be represented by the Euler integral

As

$$
\left(\begin{array}{c}
\alpha \\
i
\end{array}\right)=\frac{(-1)^{i}(-\alpha)_{i}}{i !}
$$

and attending to

$$
(i+k) !=(i+1)_{k} i !
$$

and

$$
(-\alpha)_{i+k}=(-\alpha)_{i}(-\alpha+i)_{k}
$$

we obtain

$$
R_{x}(k)=\sigma^{2}(-1)^{k} \cdot\left(\begin{array}{l}
\alpha \\
k
\end{array}\right) \cdot{ }_{2} F_{1}(\alpha,-\alpha+k ; k+1 ; 1) \quad k \geq 0
$$

Hosking [2] makes a distinction between long-term $(\alpha<0)$ and short-term $(\alpha>0)$ persistent processes, because when $\alpha<0$ the impulse response goes very slowly to zero, contrary to the $\alpha>0$ case. Note that the systems we are dealing with are the discrete-time analogue of the differintegrator in the continuous-time case. We have a differencer in the $\alpha>0$ case and an accumulator in the $\alpha<0$ case.
We define the spectrum by

$$
\begin{aligned}
S\left(\mathrm{e}^{j \omega}\right) & =\sigma^{2} \cdot \lim _{z \rightarrow \mathrm{e}^{j(\omega)}}\left(1-z^{-1}\right)^{\alpha}(1-z)^{\alpha} \\
& =\sigma^{2} \cdot\left|1-\mathrm{e}^{-j \omega}\right|^{2 \alpha} \\
& =\sigma^{2} \cdot[2 \cdot \sin (\omega / 2)]^{2 \alpha}
\end{aligned}
$$

If $\alpha>0$, the spectrum is graphically similar to a parabola. We use the term parabolic noise for the corresponding stochastic process. If $\alpha<0$, we follow Hosking's considerations to use the term hyperbolic noise, for the corresponding stochastic process, and it may be considered a fractional Brownian process, since it is generated by 'integrating' white noise. Stochastic processes of this type are the discrete-time analogue to the continuoustime 1/f noise, since near the origin $S(\omega)$ is proportional to $\omega^{2 x}$. We can show that the first difference of a hyperbolic noise is a parabolic noise. Using the relation [18]

$$
{ }_{2} F_{1}(a, b ; c ; 1)=\frac{\Gamma(c) \Gamma(c-a-b)}{\Gamma(c-a) \Gamma(c-b)} \quad c-a-b>0
$$

after some simple manipulations we obtain

$$
R_{x}(k)=\sigma^{2}(-1)^{k} \frac{\Gamma(1+2 \alpha)}{\Gamma(\alpha+k+1) \Gamma(\alpha-k+1)}
$$

leading to the power of the process:

$$
P_{x}=\sigma^{2} \frac{\Gamma(1+2 \alpha)}{[\Gamma(\alpha+1)]^{2}}
$$

if $1+2 \alpha>0$ or $\alpha>-1 / 2$. This means that, only for those values, we may be led to a stationary stochastic process. If $|\alpha|<1 / 2, x(n)$ is simultaneously stationary and invertible [2]. If $\alpha=-1 / 2$, the process has an infinite power and can be considered wide-sense stationary. This case is the most similar to the continuous-time hyperbolic noise defined previously. Asymptotically, the autocorrelation function given by eqn. 89 is proportional to $k^{-1-2 x}$, again showing hyperbolic behaviour. If $\alpha$ is positive, it goes quickly to zero, showing that the process is a short-term persistent process [2]. If $\alpha$ is negative, it is the reverse situation; it is a long-term persistent process.

An interesting result [6] refers to the partial autocorrelation function (reflection coefficient sequence). Its recursive computation with the usual Levinson-Durbin algorithm leads to

$$
C_{k}=-\frac{\alpha}{\alpha+k} \quad k=1,2, \ldots \text { and }|\alpha|<1 / 2
$$

This is an interesting result: these processes are similar to MA processes, in the sense of the similarity of the behaviours of the reflection coefficients. As seen, while the autocorrelation function is asymptotically proportional to $k^{-1-2 x}$, the partial autocorrelation is proportional to $k^{-1}$, independently of $\alpha$.

\section{Conclusions}

In this paper, we have presented a class of linear systems the fractional discrete-time systems. For the definitions of such systems, we have introduced the definitions of 'fractional delay' and 'lead'. These definitions have allowed us to present an approach that is very similar to that used in the study of the ordinary linear systems; we were led to the notions of 'fractional impulse' and 'frequency responses'. We have shown how to compute them and to test the stability that is similar to the usual systems. The problem 
of the initial conditions has also been discussed and a state variable formulation for the fractional linear time-invariant systems has been presented.

We defined the fractal stationary stochastic process as the output of a stable linear system, with the transfer function characterised by having a fractional pole or zero at $z=1$. We have discussed the concept of fractional differaccumulation, generalising the notions of 'fractal' and ' $1 / \mathrm{f}$ noise', and introduced two kinds of fractionally differaccumulated stochastic process: hyperbolic (similar to the continuous-time case) and parabolic noises.

\section{References}

1 ORTIGUEIRA, M.D.: 'Introduction to fractional signal processing Part 1: Continuous-time systems', IEE Proc. Vis., Image Signal Process., $2000,147, \mathrm{pp} .62-70$

2 HOSKING, J.R.M.: 'Fractional differencing', Biometrik, 1981, 68, (1), pp. $165-176$

3 HERMANOWICZ, E., ROJEWSKI, M., CAIN, G.D., and TARCZYNSKI: 'Special discrete-time filters having fractional delay', Signal Process., 1998, 67, pp. 279-289

4 LAAKSO, T.I., VÄLIMÄKI, V., KALJALAINEN, M., and LAINE, U.K.: 'Splitting the unit delay', IEEE Signal Process. Mag., 1996, pp. $30-60$

5 TREICHLER, J.R., FIJALKOW, I., and JOHNSON, C.R. Jr: 'Fractionally spaced equalizers: how long should they really be?', IEEE Signal Proces's. Mag., 1996, pp. 65-81

6 DE PAOR, A.M. and O'MALLEY, M.J. 'The zero-order hold equivalent transfer function for a time-delayed process', Int. J. Control, 1995 , 61. (3) pp. 657-665

7 MACHADO, J.A.T.: 'Analysis and design of fractional-order digital control systems', SAMS, 1997, 27, pp. 107-122

8 MACHADO, J.A.T, and AZENHA, A.: 'Position/force fractional control of mechanical manitpulators'. Proceedings 1998 5th Int. Workshop on Advanced Motion Control, Coimbra, Portugal, pp. 216-22

9 CRATO, $N$ : 'Recent rescarch on fractional noise in economic time series'. Proceedings 3rd CEMAPRE Conference, May 1991, Lisbon, ISEG, pp. $353-370$

10 CRATO, N.: 'Long memory time series models: a revicw'. Proceedings 3rd CEMAPRE Conference, May 1991, Lisbon, ISEG, pp. 371-390

11 DERICHE, M., and TEWFIK, A.H.: 'Signal modelling with filtered discrete fractional noise processes', IEEE Trans. Signal Process., 1993, $41,(9)$

12 JURY, E.I.: 'Theory and application of the Z-transform method' (Robert E. Krieger Publishing Co.. Huntington, New York)

13 JANSSEN A JEM. 'The Zak transform: a signal transform for JANSSEN, A.J.E.M.: 'The Zak transform: a signal transform for
sampled time-continuous signals', Philips J. Res., 1988, 43, pp. 23-69

14 MANDELBROT, B.B.: 'The fractal geometry of nature' (W. H. Freeman and Company, New York, 1983)

15 MANDELBROT, B.B., and VAN NESS, J.W: 'The fractional brownian motions, fractional noises and applications', SIAM Rev., 1968, 10, (4)

16 VAN DER ZIEL, A. 'Unified presentation of $1 / f$ noise in electronic devices: fundamental $1 / f$ noise sources', Proc. IEEE, 1988, 76, pp. 233 258

17 KESHNER, M.S.: 'l/f Noise', Proc. IEEE, 1982, 70, pp. 212-218

18 SAMKO, S.G., KILBAS, A.A., and MARICHEV, O.I.: 'Fractional integrals and derivatives - theory and application' (Grordon and Breach Science Publishers, 1987)

\section{Appendix: Cauchy problem}

Let $\boldsymbol{L}$ be the open unit circle $\left\{z: z=\mathrm{e}^{j(t)}, \omega \in[-\pi, \pi[\}\right.$. This circle decomposes the $C$ plane into two regions: the interior $C_{i}=\{z:|z|<1\}$ and the exterior $C_{e}=\{z:|z|>1\}$.

Definition $I$

We define the Cauchy problem as consisting of the determination of two functions $G^{-}$and $G^{+}$, defined and continuous over $C_{i} \cup L$ and $C_{e} \cup L$, respectively, analytic inside their domain, and satisfying

$$
G^{-}(z)+G^{+}(z)=g(z) \quad \forall z \in L
$$

We can consider $G^{-}$and $G^{+}$as extrapolations of $g(z)$ for the interior and exterior of the unit circle.

Definition 2

The integrals of the type

$$
G^{ \pm}(z)=\mp \frac{1}{2 \pi j} \int_{L} \frac{g(w)}{w-z} \mathrm{~d} w
$$

where $L$ is an integration path (closed or not), are called Cauchy integrals.

If $g(w)$ is a continuous function on $L, G^{ \pm}(z)$ is analytic in $\boldsymbol{C}-\boldsymbol{L}$ and $G^{+}(z) \rightarrow 0$ when $z \rightarrow \infty$.

The solution to the Cauchy problem is given by the Cauchy integral (eqn. 93), where the sign \pm is chosen according to the region of analyticity, $C_{e}$ or $C_{i}$. This means that we obtain two functions that may be considered as unilateral Z-transforms. However, the solution is not unique. In fact, if $A$ is a constant, the pair $G^{--}(z)-A$ and $G^{+}(z)+A$ also constitutes a solution for the problem. For our purposes, the value of $A$ must be determined according to the initial value theorem. Assume that $g(w)$, $w=\mathrm{e}^{j(s)}$, is the FT of a given signal $x_{n}$. The solution to the problem is

$$
\begin{aligned}
G^{ \pm}(z) & =\mp \frac{1}{2 \pi j} \int_{L} \frac{g(w)}{w-z} \mathrm{~d} w \pm x_{0} \\
= & \pm \frac{1}{2 \pi j} \int_{L} \frac{g(w)}{w-z} \mathrm{~d} w \pm \frac{1}{2 \pi j} \int_{L} \frac{g(w)}{w} \mathrm{~d} w \\
\text { As } \frac{1}{w-z}-\frac{1}{w} & =\frac{z}{w(w-z)}-\frac{w^{-1}}{1-w z^{-1}}: \\
G^{+}(z) & =\frac{1}{2 \pi j} \int_{L} \frac{g(w) \cdot w^{-1}}{1-w z^{-1}} \mathrm{~d} w \\
& =\frac{1}{2 \pi} \int_{-\pi}^{\pi} \frac{g\left(e^{j(\omega)}\right.}{1-e^{j(\omega)} z^{-1}} \mathrm{~d} \omega \\
\text { and } \frac{1}{w-z}-\frac{1}{w} & =\frac{z}{w(w-z)}-\frac{w^{-1} z}{w-z} \\
G^{-}(z) & =\frac{1}{2 \pi j} \int_{L} \frac{g(w) \cdot z \cdot w^{-1}}{w-z} \mathrm{~d} w \\
& =\frac{1}{2 \pi} \int_{-\pi}^{\pi} \frac{g\left(e^{j(w)}\right) \cdot z}{e^{j(\omega)}-z} \mathrm{~d} \omega \quad|z|<1
\end{aligned}
$$

By a process of analytic continuation, the function $g(z)$ defined on the unit circle $\left(z=\mathrm{e}^{j \omega x}\right)$ is extended to the region $|z|>1$ in the causal case, and $|z|<1$ in the anticausal case, in such a way that their projections on the space corresponding to that contour satisfy. eqn. 92 . 